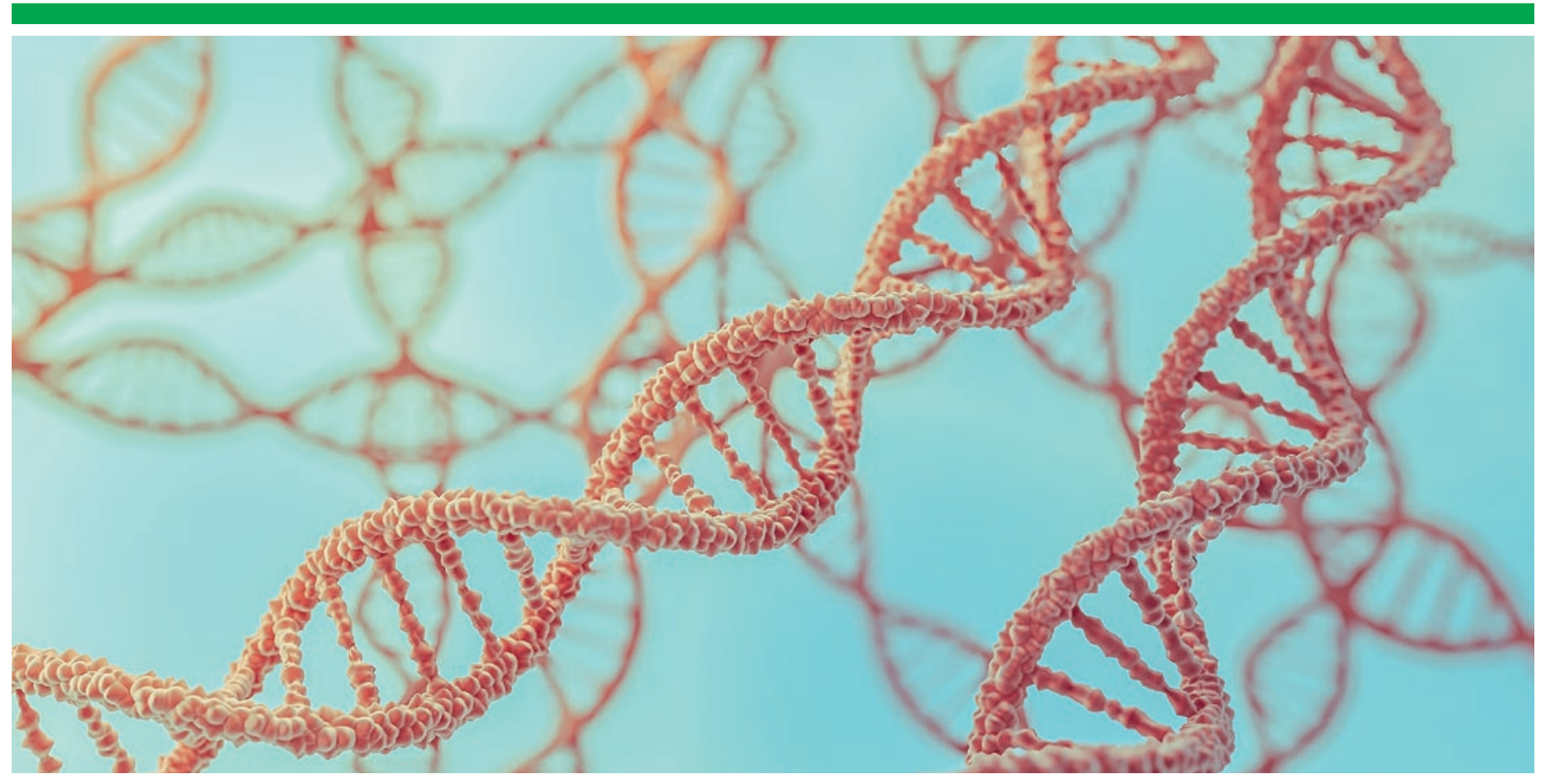

\title{
Genetische Untersuchungen im medizinischen Bereich
}

\section{Marcel Boller}

Dr. iur., Rechtsanwalt, Zürich

Das Inkrafttreten des revidierten Gesetzes über genetische Untersuchungen beim Menschen (GUMG) ist im Laufe des Jahres 2021 geplant. Revidiert wurde es vor allem aufgrund schneller technologischer und gesellschaftlicher Entwicklungen. Das Gesetz regelt die Veranlassung medizinischer genetischer Tests. Das GUMG verlangt teilweise einen Facharzttitel, damit entsprechende Untersuchungen angeordnet werden dürfen. Zudem stärkt es den Datenschutz und das Selbstbestimmungsrecht der Betroffenen, da es erhöhte Anforderungen an die Einwilligung und die Aufbewahrung der Daten stellt.

\section{Initiierung von genetischen Tests}

Genetische Tests im medizinischen Bereich sind besonders wichtig, weil sie oft mit bestimmten Therapieformen verbunden sind. Ohne entsprechende Tests könnten viele moderne Therapien nicht durchgeführt werden, da die Patientin oder der Patient bestimmte genetische Voraussetzungen erfüllen muss, damit eine Therapie erfolgreich angewendet werden kann.

Das revidierte GUMG sieht vor, dass genetische Untersuchungen im medizinischen Bereich nur durch Ärz- tinnen und Ärzte veranlasst werden dürfen. Das Gesetz enthält hierzu folgenden Grundsatz: Genetische Untersuchungen im medizinischen Bereich können von Ärztinnen und Ärzten angeordnet werden, die einen eidgenössischen Ausbildungstitel in dem Fachgebiet besitzen, dem die betreffende Untersuchung zugeordnet ist, oder die über besondere Qualifikationen in der Humangenetik verfügen [1].

Liest man diese Bestimmung, könnte der Eindruck entstehen, dass genetische Untersuchungen nur von Ärztinnen und Ärzten mit einem Facharzttitel veranlasst 
werden dürfen. Die ebenfalls revidierte Verordnung über genetische Untersuchungen beim Menschen (GUMV) stellt jedoch klar, dass nicht in allen Bereichen ein Facharzttitel erforderlich ist [2]. Ärzte der allgemeinen Inneren Medizin können auch mit Krankheiten konfrontiert werden, die Gentests erfordern. Beispielsweise erwähnt das Eidgenössische Departement des Innern (EDI) in seinen Erläuterungen ausdrücklich die Untersuchung von Volkskrankheiten wie bestimmte Gerinnungsstörungen oder familiäre Hyperlipidämie. Andere Krankheiten sind gemäss EDI ausgeschlossen: Insbesondere die Abklärung und Therapie von seltenen Krankheiten, Chromosomenstörungen oder erblich bedingten Krebserkrankungen fallen nicht in den Bereich der allgemeinen Inneren Medizin.

\section{Erfordernis eines spezifischen Facharzttitels}

In diesem Sinne wird es in Zukunft notwendig sein, genetische Tests einem bestimmten Fachgebiet zuzuordnen. Im Rahmen der Ausarbeitung der Ausführungsverordnung wurden entsprechende Listen erstellt, die eine Kategorisierung ermöglichen sollen. In Bezug auf diejenigen genetischen Untersuchungen, welche nur durch Medizinalpersonen mit entsprechendem Facharzttitel initiiert werden dürfen, hat das EDI folgende Liste entworfen:

- Untersuchung von seltenen Krankheiten, strukturellen oder numerischen Chromosomenstörungen und erblich bedingten Krebserkrankungen,

- Gentests bei Kindern und anderen urteilsunfähigen Personen (siehe Art. 16 GUMG), pränatale Gentests (siehe Art. 3 Bst. g und Art. 17 GUMG) und Gentests zur Familienplanung (siehe Art. 3 Bst. i GUMG), und

- präsymptomatische Gentests (siehe Art. 3 Bst. e GUMG).

Umgekehrt hat der Bundesrat in der revidierten GUMV auch den Versuch unternommen, bestimmte Untersuchungen ohne Facharzttitelerfordernis zu definieren. Die folgenden Prüfungen können von Ärzten veranlasst werden, die weder einen eidgenössischen Ausbildungstitel in dem Fachgebiet, dem die Prüfung zugeordnet ist, noch eine besondere Qualifikation in Humangenetik besitzen [3]:

- pharmakogenetische Studien;

- diagnostische genetische Untersuchungen, die weder seltene Krankheiten noch Chromosomenstörungen noch erblich bedingte Krebserkrankungen betreffen;

- genetische Untersuchungen, die weder seltene Krankheiten noch Chromosomenstörungen noch erblich bedingte Krebserkrankungen betreffen und sich an ein breites Publikum richten;
- genetische Untersuchungen nach Artikel 31 Absatz 1 GUMG, die zu medizinischen Zwecken erfolgen.

Trotz dieser Positivliste wird es auch in Zukunft Abgrenzungsfragen geben, da dieser Forschungsbereich einem steten und schnellen Wandel unterliegt. Daher dürfte es auch an den ärztlichen Fachorganisationen sein, noch konkretere Richtlinien zu erlassen bzw. allfällige Entwicklungen aufzunehmen und in ihren Empfehlungen an die Ärzteschaft zu implementieren.

\section{Einwilligung der Betroffenen}

Die beschriebenen genetischen Untersuchungen im medizinischen Bereich dürften häufig im Rahmen einer bestimmten Therapie oder zur Abklärung einer Therapieeignung initiiert bzw. angeordnet werden. Selbstverständlich setzt dies eine sorgfältige Abklärung der Eignung und Erforderlichkeit einer genetischen Untersuchung voraus.

Die korrekte Durchführung eines genetischen Tests durch eine Ärztin oder einen Arzt bedingt ebenso die Kenntnis und Einhaltung der datenschutzrechtlichen

Es ist sinnvoll, eine schriftliche Einwilligung einzuholen, selbst wenn die Anforderungen des Gesetzes tiefer sein sollten.

Bestimmungen, zumal der Gesetzgeber mit dem revidierten GUMG das Selbstbestimmungsrecht der Patientinnen und Patienten gestärkt hat.

In der Praxis dürfte sich dies insbesondere bei der Einholung der Zustimmung manifestieren. Das bereits im geltenden GUMG enthaltene Zustimmungserfordernis der betroffenen Person zur Durchführung einer genetischen Untersuchung stellt eines der Kernelemente zur Gewährleistung der Persönlichkeitsrechte dar. Dieses Erfordernis wird im revidierten GUMG nun dahingehend verstärkt, dass es sich um eine ausdrückliche Zustimmung des Betroffenen handeln muss [4]. Ausdrücklichkeit ist gemäss Botschaft des Bundesrates vom 5. Juli 2017 bereits gegeben, wenn eine «nonverbale Äusserung mittels eines im konkreten Kontext klaren Zeichens oder einer entsprechenden Bewegung» vorliegt.

Wie bisher sieht das revidierte Gesetz bei besonders sensitiven Untersuchungen vor, dass die Einwilligung schriftlich zu erfolgen hat [5]. Um allfällige Unsicherheiten und Haftungsfragen gleich zu Beginn auszuschliessen, ist es wohl in jedem Fall sinnvoll, eine schriftliche Einwilligung einzuholen, selbst wenn die Anforderungen des Gesetzes tiefer sein sollten. 


\section{Überschussinformationen}

Das revidierte GUMG enthält ausdrückliche Bestimmungen zu Überschussinformationen. Dabei handelt es sich um Ergebnisse einer genetischen Untersuchung, welche für den Untersuchungszweck nicht benötigt werden. Hierfür bekräftigt das revidierte GUMG das Verhältnismässigkeitsprinzip spezifisch für genetische Untersuchungen, indem Überschussinformationen grundsätzlich so weit als möglich zu vermeiden sind [6]. Konkret führt dies dazu, dass, wenn verschiedene Untersuchungsmethoden vorliegen, zwingend die Methode zu wählen ist, welche keine bzw. weniger Überschussinformationen generiert. Ebenfalls ist das untersuchende Laboratorium verpflichtet, die Medizinalperson über mögliche Überschussinformationen zu informieren [7].

Trotzdem lässt sich in vielen Fällen die Entstehung von Überschussinformationen nicht verhindern. Es gilt daher, bereits im Rahmen der Aufklärung sowohl auf die Möglichkeit der Generierung von Überschussinformationen hinzuweisen als auch auf die verschiedenen Arten von Informationen [8].

\section{Aufbewahrungsdauer von Proben und Daten}

Ausgehend vom datenschutzrechtlichen Verhältnismässigkeitsprinzip regelt das revidierte GUMG die erlaubte Dauer der Aufbewahrung für Proben und genetische Daten für den gesamten Geltungsbereich des Gesetzes [9]. Grundsätzlich dürfen Proben und Daten nur so lange aufbewahrt werden, wie dies für den damit verfolgten Zweck erforderlich ist.

- Proben und Daten im medizinischen Bereich: Es wird keine fixe Maximaldauer festgelegt, um eine möglichst hohe Anpassungsfähigkeit zu wahren.

- Proben und Daten ausserhalb des medizinischen Bereichs: Das revidierte GUMG legt die maximale Aufhebungsdauer auf 2 Jahre fest [10].

Diese Unterscheidung dürfte darauf zurückzuführen sein, dass im medizinischen Bereich Laboratorien beigezogen werden, die selbst der behördlichen Aufsicht unterstehen, wodurch eine Einhaltung der Grundsätze gewährleistet werden kann. Bei Untersuchungen ausserhalb des medizinischen Bereichs fehlt es in der Regel an einer solchen Aufsicht.

Eine längere Aufbewahrung ist in beiden genannten Fällen zulässig, sofern das Einverständnis des Betroffenen zur Verwendung für einem anderen Zweck vorliegt [11]. Dieser Vorbehalt dient der Stärkung des Rechts auf informationelle Selbstbestimmung.

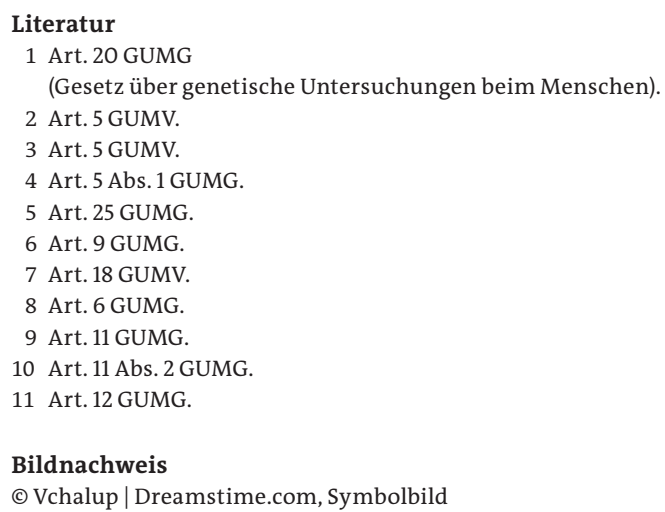

\section{L'essentiel en bref}

- Dans le courant de 2021, la Loi révisée sur les tests génétiques chez I'homme (GUMG) entrera en vigueur. Cela signifie que de nouvelles dispositions s'appliqueront dans le domaine des tests génétiques.

- Dans le cas des examens médicaux, des règles spécifiques s'appliqueront concernant le lancement de ces tests. Selon la spécialité, seuls les médecins ayant un titre de spécialiste correspondant peuvent commander des tests génétiques.

- La loi révisée renforce également la protection des données et le droit à l'autodétermination. II est donc conseillé d'obtenir à l'avenir un consentement écrit. 